\title{
Exact solution to the inverse Womersley problem for pulsatile flows in cylindrical vessels, with application to magnetic particle targeting
}

\author{
L. C. Berselli \\ Department of Applied Mathematics "U. Dini", \\ Università di Pisa, Via F. Buonarroti 1/c, Pisa, Italy \\ P. Miloro and A. Menciassi \\ The BioRobotics Institute, Scuola Superiore Sant'Anna, \\ Piazza Martiri della Libertà 33, Pisa, Italy
}

E. Sinibaldi*

Center for Micro-BioRobotics@SSSA, Italian Institute of Technology, Viale R. Piaggio 34, Pontedera, Italy

(Dated: May 15, 2012)

An exact solution to the inverse Womersley problem was derived for the fully-developed, laminar pulsatile flow of a viscous Newtonian fluid, within a cylindrical vessel with rigid walls. In particular, given an arbitrary, time-periodic flow rate, the axisymmetric velocity profile was obtained by means of two neat and computable maps relating the corresponding Fourier coefficients. The solution of such an inverse problem represents a valuable tool/benchmark for a variety of research branches, also encompassing biological fluid dynamics (including targeted drug delivery) and biomedical engineering, since flow rate is the main physical quantity which can be actually measured in many practical situations. The main advantage of the proposed analytical solution, compared to fully numerical approaches, mainly resides in computational efficiency; such an asset was demonstrated by considering both blood-like flows in larger/smaller vessels and purely pulsatile flows, which can be of interest for the development of in vitro test-benches. Moreover, the proposed solution was originally applied in the context of magnetic particle targeting, to highlight some peculiar effects on particle trajectories and capture efficiency due to pulsatility. Such a transport problem is increasingly drawing the attention of an interdisciplinary community, ranging from physicians to biomedical engineers, physicists and roboticists, thanks to its potential for targeted therapy, up to remote guidance of intravascular devices. More in general, the proposed modeling approach can be effectively exploited in a wide variety of interdisciplinary research lines.

${ }^{*}$ Correspondingauthor.Emailaddress: edoardo.sinibaldi@iit.it 


\section{INTRODUCTION}

Fully-developed, laminar flows in straight vessels have long been studied from both a theoretical and an applicative viewpoint. A fundamental, analytical solution was firstly obtained in the 19th century, independently by G.H.L. Hagen (German physicist and hydraulic engineer) and J.L.M. Poiseuille (French physician and physiologist); it described the steady flow of a viscous, incompressible fluid within a vessel having circular cross-section, and it is now well-known as Hagen-Poiseuille solution [1]. An exact, unsteady solution was then obtained by Sexl in 1930 for the pulsatile flow driven by a time-periodic pressure gradient [2]; such a solution was able to describe the so-called "annular effect" previously observed by experimentalists for some oscillatory flows (i.e. axial velocity can take a maximum near the vessel wall rather than at its center, contrarily to the case of unidirectional steady flows). Interestingly, Sexl solution is classically associated with Womersley [3], who independently re-discovered it, later in 1955. A major role in such an attribution could have been played by the instructive analysis of the relative importance between viscous and inertial effects on the resulting flow profile, as carried out by Womersley, which also led to the definition of the homonymous non-dimensional number. Anyway, in the context of theoretical fluid mechanics, a number of generalizations stemmed from Sexl work (possibly rehashed as in [4], so as to also con-

sider a constant component for the pressure gradient). In particular, more complex cross-sections were studied, either simply connected (e.g. the elliptical one, as in [5]) or not (e.g. circular and elliptical annuli, as in [6]). In addition, more complex constitutive relations were tackled, mainly by numerical approaches [7]. Further developments along both the aforementioned research directions have been continuously produced and they are still ongoing (see e.g. [8, 9]), together with extensive investigations related to complementary aspects, like flow stability (not referenced here, because they are out of the scope of the present work). As a matter of fact, Sexl/Womersley solution nowadays represents a standard benchmark for analytical/numerical methods aimed at hemodynamic investigations [10], as well as for the development of biomedical/bioengineering devices and systems [11].

Sexl/Womersley approach determines the velocity profile along the vessel radius by assuming a given, harmonic expansion for the pressure gradient. However, in most practical situations, e.g. in hemodynamics, it is extremely difficult to measure the pressure gradient, while it is affordable to measure flow rate (see e.g. [12, 13]). Consequently, it is of utmost interest to reconstruct the velocity profile by starting from a given, time-periodic flow rate, i.e. to solve the so-called inverse Womersley problem. Such a problem has been firstly addressed in [14], yet from a basic 
numerical viewpoint, while some analytical expressions for laminar pipe flows, reported in [15], have been obtained by Laplace transform technique. Moreover, additional analytical relations between the Fourier coefficients of velocity, flow rate, pressure gradient and wall shear stress have been recently presented [16]. The latter work, in particular, addresses pipes and channels flows by devoting special attention to flow reversal conditions, and it provides the aforementioned relations in terms of rather involved maps also exploiting the Bessel functions. Corresponding derivations were essentially based on the engineering approach introduced in [17], also including numerical and experimental work.

The present work focuses on the inverse Womersley problem (which is also linked with one of the nowadays classical Leray's problems [18]) by starting from a more theoretical viewpoint. Indeed, existence of a time-periodic solution for a fully-developed flow in a cylindrical vessel was only recently proved by Beirão da Veiga [19], through the accurate study of the convergence of the Fourier series defining the velocity. Moreover, a formal derivation for the map linking the flow rate Fourier coefficients to those of pressure gradient was obtained in [20], by also exploiting the results in [19] (kindly made available to the authors of [20] prior to formal publication of the technical report). Combination of latter theoretical works thus provided a fundamental existence result and constructive strategy for the inverse Womersley problem in a cylindrical vessel. In the present work, concise analytical expressions are presented, which stem from the aforementioned theoretical framework and which directly provide velocity in terms of the given flow rate. The considered expressions invoke classical Bessel and regularized confluent hyper-geometric functions, and they permit to explicitly and efficiently compute flow velocity. Such a remarkable asset is also demonstrated in the present work, by assessing the proposed analytical solution against the one obtained through a finite element analysis solver; in particular, it is associated with the very reduced computational efforts enabled by analytic treatment. Moreover, the proposed solution is originally applied in order to study some aspects of the motion of magnetoresponsive microcarriers through a pulsating, blood-like fluid. Such a transport problem is catching the attention of an interdisciplinary research community, including physicians, biomedical engineers, physicists and roboticists (see e.g. [21-29]), thanks to its potential for targeted therapy. Nonetheless, previous investigations only address transport in a steady flow: this is mainly due to the fact that magnetic drug targeting best applies in smaller vessels, where mean flow speed is mild and unsteady effects play a minor role. However, in order to effectively develop the envisaged intravascular miniature/micro devices [29], it seems reasonable to also consider relatively larger vessels, where pulsation plays a role. Hence, the exemplificative application considered in the present study takes a preliminary step towards 
the effective exploitation of analytical flow models for biomedical and microrobotic applications, and further strengthens the interdisciplinary character of the addressed research topic.

The paper is structured as follows: the inverse Womersley problem is formulated and solved in Sec. II A; Sec. IIB introduces the governing equations of the chosen application, namely magnetic particle targeting; the proposed exact solution is assessed against numerical, finite element analysis results in Sec. III A; exemplificative magnetic targeting applications are then considered in Sec. III B, with emphasis on relevant effects associated with flow pulsatility; concluding remarks are finally introduced in Sec. IV. Additional details, relevant to the magnetic targeting problem, are reported in the appendix.

\section{THEORY}

\section{A. Fluid velocity profile}

We consider a fully-developed, laminar, pulsatile fluid flow within a cylindrical vessel $V:=$ $S \times \mathcal{H}$ having circular cross-section $S$ with radius $R>0$ (cylinder length is irrelevant, hence it is unnecessary to specify the corresponding non-empty interval $\mathcal{H}$ ). More precisely, we assume that: (i) the flow is governed by the incompressible Navier-Stokes equations, coupled with the classical no-slip boundary condition [30]

$$
\begin{cases}\nabla \cdot \mathbf{v}=0 & \text { in } V \times \mathcal{T} \\ \partial_{t} \mathbf{v}+(\mathbf{v} \cdot \nabla) \mathbf{v}-\nu \Delta \mathbf{v}+\frac{1}{\rho} \nabla p=0 & \text { in } V \times \mathcal{T} \\ \mathbf{v}=0 & \text { on } \partial S \times \mathcal{H} \times \mathcal{T}\end{cases}
$$

where $\mathbf{v}$ denotes fluid velocity, $p$ is pressure, $\rho>0$ and $\nu>0$ respectively represent fluid (constant) density and kinematic viscosity, and $\mathcal{T}$ denotes a chosen time-interval; (ii) the volumetric flow rate $q(t)$ is assigned as a time-periodic function with period $T$; (iii) in a Cartesian frame having the $z$-axis aligned with the cylinder axis (so that $x$ and $y$ span $S$ ), $\mathbf{v}$ is directed along $z$ and does not depend on $z$, namely $\mathbf{v}=v(x, y, t) \hat{\mathbf{e}}_{z}$. Below we provide an explicit and computable expression for $v$, depending on the adopted flow rate $q$ (besides geometrical and physical data). It should be noticed that assigning the flow rate makes the considered problem non-standard. Indeed, one has to reconstruct $\mathbf{v}$ and $p$ from $q$ by solving an inverse problem $[18,20]$, differently from classical 
approaches where velocity and pressure are obtained from the initial data, through the solution of an initial boundary value problem.

Thanks to circular symmetry, problem (1) is best reformulated by introducing the radial coordinate $r:=\sqrt{x^{2}+y^{2}}$ in place of $x$ and $y$, such that $S=\{r<R\}$ and $v=v(r, t)$. Moreover, once substituted the latter expression into the Navier-Stokes equations, it turns out that $p$ does not depend on $r$, and $\partial_{z} p$ does not depend on $z$; hence, it is possible to define $\sigma(t):=-\rho^{-1} \partial_{z} p(z, t)$. In light of the above positions, $v$ and $\sigma$ are the solutions of the following problem in the vessel cross-section $S$ :

$$
\begin{cases}\partial_{t} v(r, t)-\nu \Delta v(r, t)=\sigma(t) & \text { in } S \times[0, T], \\ v=0 & \text { on } \partial S \times[0, T],\end{cases}
$$

further subjected to the following constraint:

$$
2 \pi \int_{S} v(r, t) r \mathrm{~d} r=q(t), \quad t \in[0, T] .
$$

The considered system of equations generalizes the classical problems studied by Hagen and Poiseuille in the stationary case [31], and by Sexl/Womersley in the time-dependent case [2, 3]. Indeed, for a constant flow rate $q(t)=q_{0}$ it is straightforward to recover the well-known, parabolic Hagen-Poiseuille solution $v(r, t)=v_{0}(r)$, with

$$
v_{0}(r):=\frac{\sigma_{0} R^{2}}{4 \nu}\left[1-\left(\frac{r}{R}\right)^{2}\right], \quad \sigma_{0}:=\frac{8 \nu q_{0}}{\pi R^{4}} .
$$

Moreover, Sexl/Womersley solution can be recovered by neglecting the flow rate constraint in Eq. (3), and by assuming that pressure gradient $\sigma$ is assigned, of the form $\sigma=\tilde{\sigma} e^{j \omega t}$, where $\tilde{\sigma}$ is a reference value, $\omega=2 \pi / T$ denotes the enforced flow pulsation and $j$ is the imaginary unit $\left(j^{2}=\right.$ -1 ). The solution at hand is more involved than Hagen-Poiseuille one, since it passes through the solution of an ordinary differential equation of the type $u^{\prime \prime}(r)+u^{\prime}(r) / r-j \omega u(r) / \nu=-\tilde{\sigma} / \nu$ (prime denoting differentiation), which however can be explicitly obtained in terms of Bessel functions. A major contribution due to Womersley was to show that resulting flow profile essentially depends on the non-dimensional parameter

$$
\text { Wo }:=R \sqrt{\frac{\omega}{\nu}}
$$

thereafter called Womersley number.

As above claimed, in the present study we consider an inverse problem where the flow rate $q$ is given, and the two unknowns are $v$ and $\sigma$. More precisely, in order to study the considered 
time-periodic problem, we assume the following Fourier expansions

$$
\left(\begin{array}{c}
q(t) \\
\sigma(t) \\
v(r, t)
\end{array}\right)=\sum_{n=-N}^{N}\left(\begin{array}{c}
q_{n} \\
\sigma_{n} \\
v_{n}(r)
\end{array}\right) e^{j \omega_{n} t}
$$

where $q_{n}, \sigma_{n}, v_{n} \in \mathbf{C}, N \in \mathbf{N}$ is a chosen parameter and $\omega_{n}:=2 \pi n / T$; we hereafter introduce computable expressions for the Fourier modes $v_{n}$ and $\sigma_{n}$ in terms of $q_{n}$. Our constructive strategy, grounded on the basic existence result recently obtained in [19], works as follows: assuming a given pressure gradient, we firstly evaluate $v_{n}$ in terms of some unknown $\sigma_{n}$ as in [3]; we then compute the flow rate associated with $v_{n}$ and impose it to be equal to $q_{n}$. It is worth emphasizing that: (i) subsequent calculations can be carried out in a completely explicit manner, at least in terms of some special functions as in Eq. (7) and Eq. (11), still thanks to circular symmetry; (ii) the proposed method can be also applied when considering an infinite expansion for the flow rate $q$, since the problem is linear and the Fourier modes are decoupled. Nevertheless, in view of practical applications, a finite expansion is considered here, because the flow rate is experimentally recorded and it is typically decomposed into a finite number of Fourier modes; (iii) we can study the relation amongst $q_{n}, \sigma_{n}$ and $v_{n}$ by assuming $n>0$. Indeed, the case $n=0$ is consistently provided by Eq. (4). Moreover, since we are interested in real valued solutions, we directly impose $\left(q_{-n}, \sigma_{-n}, v_{-n}\right)=\left(q_{n}^{\star}, \sigma_{n}^{\star}, v_{n}^{\star}\right)$, where star superscript denotes complex conjugation.

We firstly considered the $q_{n} \mapsto \sigma_{n}$ map, which was formally studied in [20] for an arbitrary crosssection, through the solution of two coupled Poisson problems (or a single fourth-order equation) for each Fourier mode. We were able to explicitly solve the aforementioned Poisson problems for the circular cross-section $S$ considered in the present study, so as to obtain the following map:

$$
\frac{q_{n}}{\pi R^{2}}=\left[1-\frac{{ }_{0} \tilde{F}_{1}\left(; 2 ; j \mathrm{Wo}_{R, n}^{2} / 4\right)}{{ }_{0} \tilde{F}_{1}\left(; 1 ; j \mathrm{Wo}_{R, n}^{2} / 4\right)}\right] \frac{\sigma_{n}}{j \omega_{n}}, \quad n>0,
$$

where

$$
{ }_{0} \tilde{F}_{1}(; b ; w):=\sum_{k=0}^{\infty} \frac{w^{k}}{k ! \Gamma(b+k)}, \quad b, w \in \mathbf{C},
$$

denotes the regularized confluent hyper-geometric (limit) function, $\Gamma$ representing the Euler gamma function given by

$$
\Gamma(w):=\int_{0}^{\infty} \tau^{w-1} e^{-\tau} d \tau, \quad w \in \mathbf{C} .
$$

Furthermore, once defined a sort of generalized Womersley number as follows:

$$
\mathrm{Wo}_{r, n}:=r \sqrt{\frac{\omega_{n}}{\nu}}
$$


the non-dimensional parameter $\mathrm{Wo}_{R, n}$ in Eq. (7) is obtained by evaluating $\mathrm{Wo}_{r, n}$ in correspondence of $r=R$. For the sake of completeness, we mention that in [20] the sought map was formally obtained by considering a Fourier expansion with sines and cosines, yet conversion into complex exponential form is straightforward (see also Sec. IIIA). The $\sigma_{n} \mapsto v_{n}$ map was then obtained by integrating over the vessel cross-section; once evaluated the integrals in polar coordinates, the following relation was obtained

$$
v_{n}=\left\{1-\frac{J_{0}\left[(-1)^{3 / 4} \mathrm{Wo}_{r, n}\right]}{J_{0}\left[(-1)^{3 / 4} \mathrm{Wo}_{R, n}\right]}\right\} \frac{\sigma_{n}}{j \omega_{n}}, \quad n>0,
$$

where

$$
J_{k}(w):=\sum_{m=0}^{\infty} \frac{(-1)^{m}(w / 2)^{2 m+k}}{m ! \Gamma(m+k+1)}, \quad k \in \mathbf{N}, w \in \mathbf{C},
$$

denote the Bessel functions of the first kind, with $\Gamma$ and $\mathrm{Wo}_{r, n}$ respectively defined in Eq. (9) and Eq. (10). In particular, Eq. (11) is consistent with the ones reported in literature for the direct Womersley problem.

In summary, the proposed model is constructed as follows: (i) the given flow rate $q(t)$ is decomposed into Fourier modes $\left(q_{-N}, \ldots, q_{-1}, q_{0}, q_{1}, \ldots, q_{N}\right)$; (ii) $q_{0}$ directly provides $\sigma_{0}$ and $v_{0}$ through Eq. (4); (iii) for $n>0, \sigma_{n}$ is obtained by inverting Eq. (7) and $v_{n}$ is subsequently obtained through Eq. (11); (iv) $\sigma_{-n}$ and $v_{-n}(n=1, \ldots, N)$ are respectively obtained as complex conjugate of $\sigma_{n}$ and $v_{n}$; (v) finally, $\sigma(t)$ and $v(t)$ are obtained by summation, as in Eq. (6). We conclude the present section by remarking that theoretical results in $[19,20]$ were invoked to be sure that the solution to the problem of finding $v_{n}$ given $q_{n}$ exists. Then, the elegant and explicit maps reported in Eq. (7) and Eq. (11) were consistently derived; the former, in particular, originally appears in the present work.

\section{B. Magnetic particle targeting}

By recalling the analytical model introduced in Sec. II A, we address the effect of pulsation on the capture of an isolated magnetoresponsive microcarrier by means of an external magnetic field. Such a problem is well studied in literature, in the context of magnetic drug targeting, yet under the simplifying assumption of steady flows within small diameter vessels (see e.g. [21-23, 25-28]). In view of such a focused objective, we assume for simplicity that: (i) considered microcarrier can be described as a spherical particle having radius $R_{p}$ (particle and carrier are hereafter used as synonyms); (ii) particle size is large enough for Brownian effects to be negligible (see e.g. [21] 
for relevant conditions), at the same time it is small enough not to affect the carrying flow; (iii) magnetic force $\mathbf{F}_{m}$ and fluidic force $\mathbf{F}_{d}$ due to viscous drag are the only relevant forces acting on the particle (i.e. inertial effects and other forces like gravity/buoyancy or lift due to velocity gradients in the flow play a minor role, see e.g. [25] for relevant conditions). Based on these assumptions, we describe the motion of an isolated magnetoresponsive particle by adopting a Newtonian approach, for which equilibrium simply reads $\mathbf{F}_{m}+\mathbf{F}_{d}=\mathbf{0}$.

As regards fluidic force, we adopt the classical Stokes model $\mathbf{F}_{d}=-6 \pi \mu R_{p}\left(\mathbf{v}_{p}-\mathbf{v}\right)$, where $\mu>0$ denotes fluid (dynamic) viscosity, while $\mathbf{v}_{p}$ and $\mathbf{v}$ respectively represent particle and fluid velocity. It is worth mentioning that, for practical applications, particle radius $R_{p}$ should be replaced with an effective hydrodynamic radius (possibly accounting for surface-bound material [24]), yet such a refinement is here neglected for simplicity. The considered viscous drag model is consistent with the fact that, for typical values of the involved parameters, particle Reynolds number is $\operatorname{Re}_{p}:=2 R_{p}\left|\mathbf{v}_{p}-\mathbf{v}\right| / \nu \ll 1[21,25]$.

As regards magnetic force, we adopt an established point-dipole approach (see e.g. [21-25, 27]) according to which $\mathbf{F}_{m}=\mu_{0}\left(1+\chi_{f}\right) \beta_{m} V_{p} f(H)(\mathbf{H} \cdot \nabla) \mathbf{H}$, where $\mu_{0}=4 \pi \cdot 10^{-7} \mathrm{Tm} / \mathrm{A}$ is vacuum magnetic permeability, $\chi_{f}$ denotes fluid magnetic susceptibility, $V_{p}=4 \pi R_{p}^{3} / 3$ is particle volume, and $\mathbf{H}$ represents the externally applied magnetic field at the particle center. Furthermore, parameter $\left.\left.\beta_{m} \in\right] 0,1\right]$ defines the fraction of the carrier volume $V_{p}$ which effectively contributes to the magnetic response, e.g. as in [23]. Moreover, demagnetization effects and particle saturation are accounted for, through the function $f(H)$ defined as follows:

$$
f(H):= \begin{cases}\phi_{p, f} \quad \text { if } M_{p}^{s} / H>\phi_{p, f}, \\ M_{p}^{s} / H \quad \text { if } M_{p}^{s} / H \leq \phi_{p, f},\end{cases}
$$

where $\phi_{p, f}:=3\left(\chi_{p}-\chi_{f}\right) /\left[\left(\chi_{p}-\chi_{f}\right)+3\left(1+\chi_{f}\right)\right]$, while $\chi_{p}$ and $M_{p}^{s}$ denote particle magnetic susceptibility and saturation magnetization, respectively.

In light of the aforementioned positions, particle trajectory is determined by integrating the following ordinary differential equation:

$$
\mathbf{v}_{p}=\mathbf{v}+\zeta f(H)(\mathbf{H} \cdot \nabla) \mathbf{H}
$$

where $\zeta:=2 \mu_{0}\left(1+\chi_{f}\right) \beta_{m} R_{p}^{2} /(9 \mu)$, and starting from a suitable initial condition. Clearly, by defining $\mathbf{v}$ through the analytical flow model introduced in Sec. II A, it is possible to effectively exploit the considered formulation for unsteady problems as well, so as to study magnetic targeting in a more general context. 
TABLE I: Parameters of the test-cases introduced to assess the proposed analytical model for pulsatile flows: vessel radius $(R)$, speed associated with time-averaged flow rate $(\bar{v})$, speed associated with oscillating component of the flow rate $(\delta v)$, number of Fourier modes $(N)$, and corresponding Fourier coefficients $\left(a_{n}\right.$, $b_{n}$, with $\left.n=1, \ldots, N\right)$.

\begin{tabular}{cccccll}
\hline \hline Test-case & $\begin{array}{c}R \\
(\mathrm{~mm})\end{array}$ & $\begin{array}{c}\bar{v} \\
(\mathrm{~cm} / \mathrm{s})\end{array}$ & $\begin{array}{c}\delta v \\
(\mathrm{~cm} / \mathrm{s})\end{array}$ & $\mathrm{N}$ & $\left(a_{1}, \ldots, a_{N}\right) \cdot 10$ & $\left(b_{1}, \ldots, b_{N}\right) \cdot 10$ \\
\hline TC-1a & 8 & 10 & 5 & 2 & $(2,7)$ & $(1,4)$ \\
TC-1b & 8 & 10 & 5 & 8 & $(2,0.7,0,0.6,-2,-2,1,1)$ & $(1,4,-0.5,1,0.4,0,-2,-1)$ \\
TC-2a & 8 & 1 & 0.2 & 2 & $(-3,4)$ & $(0,6)$ \\
TC-2b & 8 & 1 & 0.2 & 8 & $(0.2,0.7,-2,0,0.9,-1,-3,0.5)$ & $(1,0.4,-5,1,7,-0.1,0.4,0)$ \\
TC-3a & 2 & 0.1 & 0.005 & 2 & $(4,1)$ & $(4,-4)$ \\
TC-3b & 2 & 0.1 & 0.005 & 8 & $(0.3,0.1,-4,2,0.3,-1,-0.3,0)$ & $(2,0.1,-2,0.1,0.7,-1,4,3)$ \\
TC-4a & 2 & 0 & 0.2 & 2 & $(1,4)$ & $(2,7)$ \\
TC-4b & 2 & 0 & 0.2 & 8 & $(0.2,0.7,-0.1,3,1,0.4,-0.9,4)$ & $(0.7,0.4,-1,0.5,1,-0.4,2,1)$ \\
\hline \hline
\end{tabular}

\section{RESULTS}

\section{A. Fluid velocity profile}

In this section we compare the results obtained by using the analytical model introduced in Sec. II A with those obtained by a commercially available finite element analysis (FEA) solver. In particular, we compute the velocity profile in a circular cylindrical vessel, under several flow conditions summarized in Table I. For all the considered test-cases the assumed flow period is $T=$ $1 \mathrm{~s}$. More in detail, we consider a given flow rate of the form

$$
q(t)=\bar{q}+\delta q \cdot \sum_{n=1}^{N}\left[a_{n} \sin \left(\omega_{n} t\right)+b_{n} \cos \left(\omega_{n} t\right)\right],
$$

where time-averaged flow rate and flow rate oscillating component are respectively given by $\bar{q}:=\bar{v} \pi R^{2}$ and $\delta q:=\delta v \pi R^{2}$, with $R, \bar{v}$ and $\delta v$ assigned in Table I. It is evident that testcases TC- $\lambda \mathrm{a}$ and $\mathrm{TC}-\lambda \mathrm{b}(\lambda \in\{1,2,3,4\})$ only differ from each other as for the oscillating harmonics content. Relevant Fourier coefficients $a_{n}, b_{n} \in \mathbf{R}$ are also listed in Table I; they can be straightforwardly related to the complex ones in Eq. (6) as follows: $q_{0}=\bar{q}, q_{n}=\delta q\left(b_{n}-j a_{n}\right) / 2$, $q_{-n}=\delta q\left(b_{n}+j a_{n}\right) / 2$, for $n=1, \ldots, N$. Some parameters have been chosen as representative of blood flows in humans. In particular, $R=8 \mathrm{~mm}$ is intended to be representative of larger arteries: corresponding diameter is, for instance, in between the one of abdominal aorta (say 20 to $25 \mathrm{~mm}$ ) and that of carotid arteries (say $5 \mathrm{~mm}[32]$ ). For the aforementioned arteries, mean flow speed is 
of the order of $10 \mathrm{~cm} / \mathrm{s}$, and the difference between peak and average blood speed is of the same order of the average speed itself [12]. Hence, flow rate associated with test-case TC-1 (here and below suffixes $a$ and $b$ are tacitly understood) can be regarded to as representative of physiological conditions in human larger arteries. Test-case TC-2, instead, can describe non-physiological flow conditions in larger vessels, since it involves the same vessel diameter as TC-1 and a reduced flow rate. Conversely, flow reduction physiologically occurs in smaller vessels, where unsteady effects due to pulsation also become negligible [33]. Test-case TC-3 consistently addresses a very-low Reynolds number flow, while keeping some harmonics content (fully neglecting pulsation effects, indeed, is not of interest in the present context). Finally, test-case TC-4 does not address physiological flow conditions. It aims at assessing model ability to describe purely pulsatile flows (i.e. with a null average flow rate), where also backflow (potentially occurring, at least locally, in the previous test-cases) plays an important role. Nonetheless, such flow condition can be of interest for developing in vitro test-benches.

In order to quantitatively label the above discussed test-cases, we introduce the following Reynolds and Womersley numbers:

$$
\operatorname{Re}:=\frac{(\bar{v}+\delta v) R}{\nu}, \quad \mathrm{Wo}_{R, N}=R \sqrt{\frac{\omega_{N}}{\nu}}
$$

where $\mathrm{Wo}_{R, N}$ is derived from expression (10). Corresponding values are reported in Table II, as obtained by assuming $\nu=3.4 \mathrm{~mm}^{2} / \mathrm{s}$. Such value is associated with human plasma at physiological temperature (density is similar to water, dynamic viscosity is nearly 3.5 times larger than that of water), and it is also representative for blood (with the exception of tiny vessels, having diameter below $500 \mu \mathrm{m}$, where hematocrit concentration plays a role [21]). This choice is consistent, in particular, with the flow conditions adopted for test-cases TC-1 to TC-3. It is worth remarking that, by virtue of the considered values of Re, the flows at hand are expected to be laminar. Indeed, the laminar/turbulent transition Reynolds number for steady pipe flows is around 2000 (may be higher, based on flow quality); for pulsatile flows such a threshold also depends on pulsation amplitude and frequency. However, adopting a pulsation amplitude such that maximum Reynolds number in the vessel (which is roughly estimated by the above definition of Re) does not exceed 2000 would guarantee a laminar pulsatile flow [16].

Exemplificative results obtained by exploiting the proposed analytical model are reported in Fig. 1-4, together with the corresponding results provided by the commercial FEA solver COMSOL Multiphysics (CM) by Comsol Inc., available to the group. As regards CM simulations, once 
TABLE II: Reynolds number and Womersley number of the test-cases introduced in Table I.

\begin{tabular}{ccc}
\hline \hline Test-case & Re & $\mathrm{Wo}_{R, N}$ \\
\hline TC-1a & 351.43 & 15.35 \\
TC-1b & 351.43 & 30.69 \\
TC-2a & 28.11 & 15.35 \\
TC-2b & 28.11 & 30.69 \\
TC-3a & 0.61 & 3.84 \\
TC-3b & 0.61 & 7.67 \\
TC-4a & 1.17 & 3.84 \\
TC-4b & 1.17 & 7.67 \\
\hline \hline
\end{tabular}

assigned the relevant physical parameters, vessel radius was defined for each test-case based on the values in Table I. Moreover, a finite-length domain was considered, yet length-to-radius ratio was set large enough (namely 20 to 80 ) to damp inlet and outlet boundary effects, thus allowing for a fully-developed flow at least in the central portion of the domain (such a condition was $a$ posteriori checked). Furthermore, the flow rate specified by Eq. (15) was imposed (by software coding) at the inlet cross-section, the no-slip boundary condition was enforced at vessel wall, and a reference pressure value was imposed at the outlet cross-section (such value is immaterial, due to the incompressible formulation). Moreover, cylindrical symmetry was directly enforced by exploiting the axisymmetric solver available within CM: this choice permitted to save computational time, not only during the actual runs but also during the simulation set-up phase. Finally, as regards simulation set-up, both space- and time-discretization were incrementally refined, up to obtain discretization-independent results. Thirty pulsation periods were then simulated, so as to get timeperiodic numerical results after a certain transient (which took up to ten periods). All simulations were run on a single core of a PC with the following characteristics: Intel Xeon E5420 $2.50 \mathrm{GHz}$ CPU, 2x6 MB L2 cache, 8 GB RAM. Discrepancy between analytical and FEA velocity profiles was measured by introducing a relative error $\epsilon$, normalized through the characteristic speed $\bar{v}+\delta v$. A very good matching was obtained: $\epsilon<1.26 \%$ for TC-1 and $\epsilon<0.13 \%$ for TC-2 to TC-4 (for all values of $r / R \in[0,1]$ and $t / T \in[0,1])$. Such an agreement is consistent with the fact that both approaches are based on the same PDE problem. Nevertheless, it may be worth remarking that the proposed model can also describe rather complex flows, e.g. involving considerable backflow as in TC-4 (minor backflow also occurred for e.g. TC-1, close to the vessel wall). On the other hand, the main strength of the proposed analytical approach resides in direct computability, leading in 


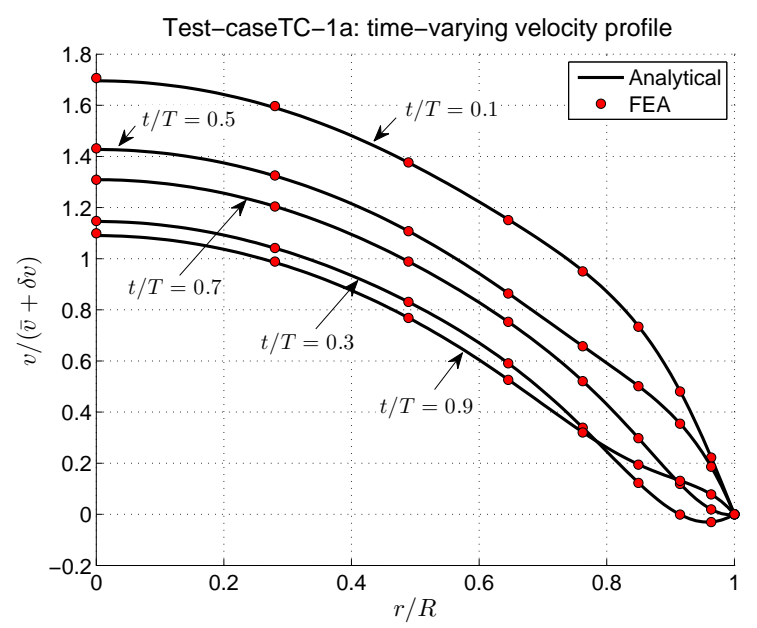

FIG. 1: (Color online) Flow velocity profile for test-case TC-1a, as obtained by the proposed analytical method (solid curve) and by FEA (filled circles). Normalized profile $v(r, t) /(\bar{v}+\delta v)$ is plotted against $r / R \in[0,1]$, for the following non-dimensional times $t / T$ within a period: $\{0.1,0.3,0.5,0.7,0.9\}$.

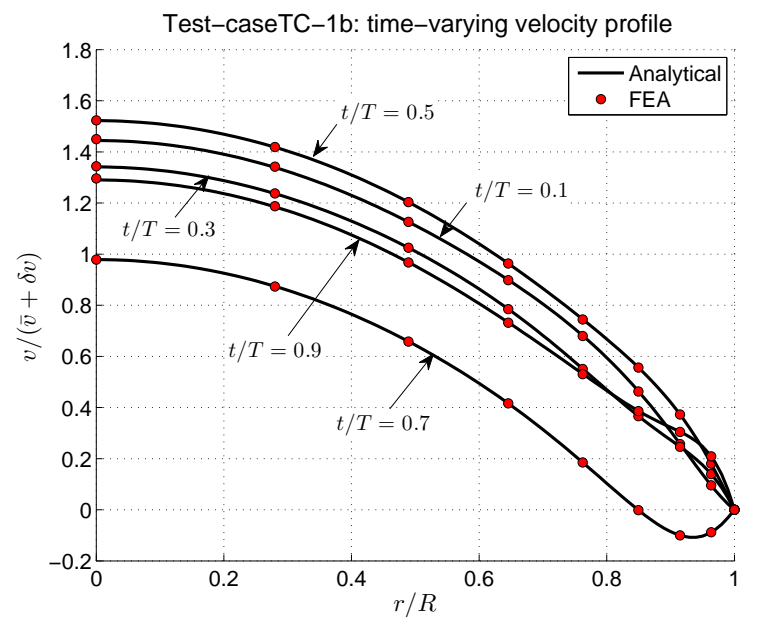

FIG. 2: (Color online) Flow velocity profile for test-case TC-1b, as obtained by the proposed analytical method (solid curve) and by FEA (filled circles). Normalized profile $v(r, t) /(\bar{v}+\delta v)$ is plotted against $r / R \in[0,1]$, for the following non-dimensional times $t / T$ within a period: $\{0.1,0.3,0.5,0.7,0.9\}$.

particular to reduced computational times. Indeed, CPU time for the considered FEA simulations ranged from roughly $3.5 \mathrm{~h}$ for TC-2 and TC-3, to $13.5 \mathrm{~h}$ for TC-1a, up to nearly $20 \mathrm{~h}$ for TC-1b. Larger CPU times for TC1 were essentially due to the increased average flow speed, also requiring a longer computational domain for a fully-developed flow to be obtained. Moreover, TC-1b required an increased CPU time compared to TC-1a due to enhanced unsteady effects, associated with its richer harmonics content. This aspect was even more pronounced for TC-4b versus TC-4a (please 


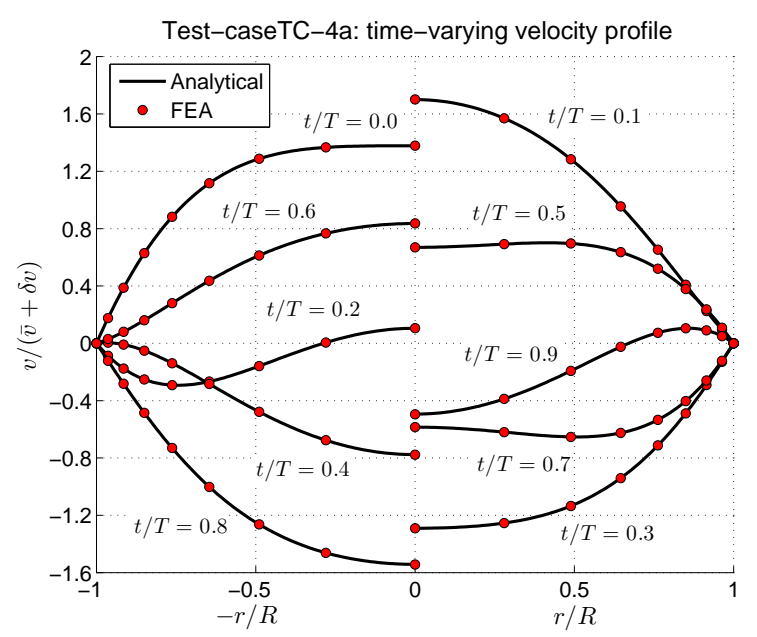

FIG. 3: (Color online) Flow velocity profile for test-case TC-4a, as obtained by the proposed analytical method (solid curve) and by FEA (filled circles). Normalized profile $v(r, t) /(\bar{v}+\delta v)$ is plotted against $r / R \in[0,1]$, for the following non-dimensional times $t / T$ within a period: $\{0.1,0.3,0.5,0.7,0.9\}$. Moreover, for ease of representation, the considered normalized velocity profile is also plotted against $-r / R \in[-1,0]$, for the following non-dimensional times: $\{0.0,0.2,0.4,0.6,0.8\}$.

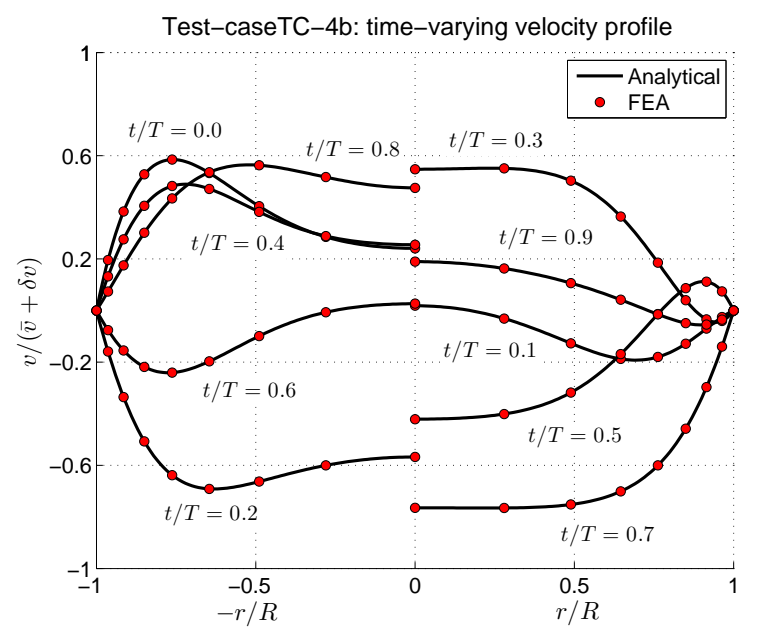

FIG. 4: (Color online) Flow velocity profile for test-case TC-4b, as obtained by the proposed analytical method (solid curve) and by FEA (filled circles) [34]. Normalized profile $v(r, t) /(\bar{v}+\delta v)$ is plotted against $r / R \in[0,1]$, for the following non-dimensional times $t / T$ within a period: $\{0.1,0.3,0.5,0.7,0.9\}$. Moreover, for ease of representation, the considered normalized velocity profile is also plotted against $-r / R \in[-1,0]$, for the following non-dimensional times: $\{0.0,0.2,0.4,0.6,0.8\}$.

notice the many and more pronounced extrema in Fig. 4 compared to Fig. 3): TC-4a took 3.5 h, while TC-4b required 7.5 h. Furthermore, set-up time for a generic run (namely an estimate of the time spent to firstly achieve a discretization-independent, periodic, fully-developed flow) was in 
the order of 10 to $20 \mathrm{~h}$. Conversely, it took less than $1 \mathrm{~h}$ to set-up the analytical approach within Matlab rapid code prototyping environment and, most importantly, CPU time was in the order of a few seconds for all the considered test-cases. Hence, computational efficiency (by no means to be negotiated with accuracy) clearly represents a most remarkable asset of the proposed analytical model.

\section{B. Magnetic particle targeting}

In order to illustrate how the introduction of pulsatile flow profiles leads to a richer repertoire of magnetic particle trajectories, we considered the magnetic targeting setup sketched in Fig. 5. In

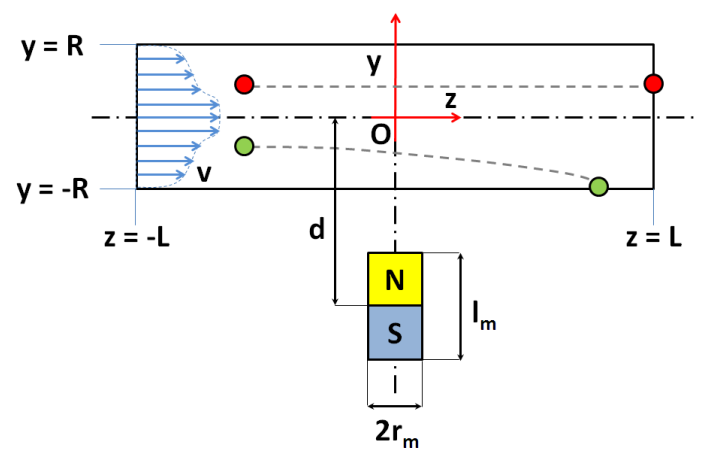

FIG. 5: (Color online) Setup considered for magnetic particle targeting (schematic). Vessel axis perpendicularly intersects the axis of an external, cylindrical magnet, so as to define a Cartesian frame. Velocity profile is pictorially sketched (arrows) at inlet, together with the trajectories (dashed lines) of two particles (circles): the one closer to the magnet is captured, while the other escapes from outlet. Symbols N and S respectively denote north and south poles of the magnet. Plane $y z$ is only sketched, for ease of representation.

the considered setup, the external magnetic field is produced by a permanent, axially magnetized cylinder, whose axis perpendicularly intersects vessel axis so as to define the Cartesian frame shown in the figure at hand. A relevant model for the considered magnetic field is recalled in the appendix, for ease of presentation; relevant parameters for such model are cylinder radius $r_{m}$, cylinder length $l_{m}$ and saturation magnetization $M_{m}^{s}$. Moreover, the distance between vessel axis and magnet center is denoted by $d$ in Fig. 5, while $L$ denotes vessel half-length; the latter was chosen large enough for magnetic force to be negligible at both inlet and outlet sections.

The following parameters were adopted: $\rho=1000 \mathrm{~kg} / \mathrm{m}^{3}, \nu=3.5 \mathrm{~mm}^{2} / \mathrm{s}$ (so that $\mu=3.5 \mathrm{mPa}$ s), $R=3.5 \mathrm{~mm}, \bar{v}=10 \mathrm{~cm} / \mathrm{s}, \chi_{f}=0$ and $\phi_{p, f}=3$ (since for water-/blood-like fluids and commonly used magnetic materials like magnetite $\chi_{f} \ll 1 \ll \chi_{p}$ ), $R_{p}=1.5 \mu \mathrm{m}, \beta_{m}=1$ (for simplicity), $M_{p}^{s}=$ 
$0.5 \mathrm{MA} / \mathrm{m}$ (as for magnetite), $r_{m}=4 \mathrm{~cm}, l_{m}=8 \mathrm{~cm}, M_{m}^{s}=1 \mathrm{MA} / \mathrm{m}$ (as for common NdFeB magnets), $d=4.5 \mathrm{~cm}$. Some of the above parameters were directly adopted from [21, 25], in order to also legitimate the assumptions introduced in Sec. IIB (and, consequently, to adopt Eq. (14) as governing equation for particle trajectory). Finally, a pulsatile component with period $T=1 \mathrm{~s}$ was added to the average flow introduced above, so as to consider the effect of unsteadiness on particle targeting. More in detail, with reference to the definitions introduced in Sec. III A, the following parameters were adopted: $\delta v=5 \mathrm{~cm} / \mathrm{s}, N=8,\left(a_{1}, \ldots, a_{8}\right) \cdot 10=(4,6,0,4,2,0,0.7,0.1)$, $\left(b_{1}, \ldots, b_{8}\right) \cdot 10=(5,-5,5,1,0.4,0,2,1)$.

Exemplificative magnetic particle trajectories are reported in Fig. 6 and Fig. 7. In particular, particles considered in Fig. 6 were seeded at non-dimensional time $t_{0} / T=0.5$ (hereafter subscript 0 denotes initial conditions) on the symmetry plane shown in Fig. 5 , namely at $x_{0} / R=0, z_{0} / L=-1$ and by varying $y_{0} / R$. Dashed lines - associated with empty markers - show the trajectories in the steady case (defined by only considering the average speed $\bar{v}$ ), while solid lines - associated with filled markers - represent the corresponding unsteady trajectories (obtained by also considering $\delta v)$. It can be seen that there may exist a region where pulsation effects dramatically affect trajectories: some of the particles captured (i.e. led to the vessel boundary) in the steady case are allowed to escape in the unsteady one, and vice versa. Interestingly, the considered figure also shows that, in the unsteady case, some particles may be captured even if others which were seeded closer to the magnet cannot. This result substantially differs from the steady case, where the efficacy of the magnetic link on capture is only determined by spatial factors (in particular, it monotonically decreases when the seeding point is moved farther away from the magnet). In particular, such a peculiar result seems to be associated with the annular effect, since flow velocity is not monotonically decreasing towards the vessel walls.

Clearly, when considering pulsatile flow conditions, particle seeding time is expected to play a role in magnetic targeting. This is confirmed, for instance, by Fig. 7, where particles were seeded (still at $x_{0} / R=0, z_{0} / L=-1$ and by varying $y_{0} / R$ ) at two different non-dimensional times $t_{0} / T$, namely 0.8 (solid lines, with filled markers) and 0.9 (dashed lines, with empty markers). Incidentally, for the latter seeding time all considered particles can be captured (even the one starting from $y_{0} / R=-0.4$, which conversely escapes when seeded at $t_{0} / T=0.5$, according to Fig. 6 ).

A figure of merit for therapeutic applications of particle targeting is capture efficiency. Given $n_{\text {in }}$ particles uniformly seeded at the inlet section, $n_{\text {out }}$ of which also escape from the outlet, capture efficiency is defined as follows: $\eta:=\left(n_{\text {in }}-n_{\text {out }}\right) / n_{\text {in }}$. In the steady case, the ratio $\mathrm{Mn}_{p}:=F_{m 0} / F_{d 0}$ between the intensities of magnetic force $F_{m 0}$ and drag force $F_{d 0}=6 \pi \mu R_{p}(2 \bar{v})$ for a steady particle 


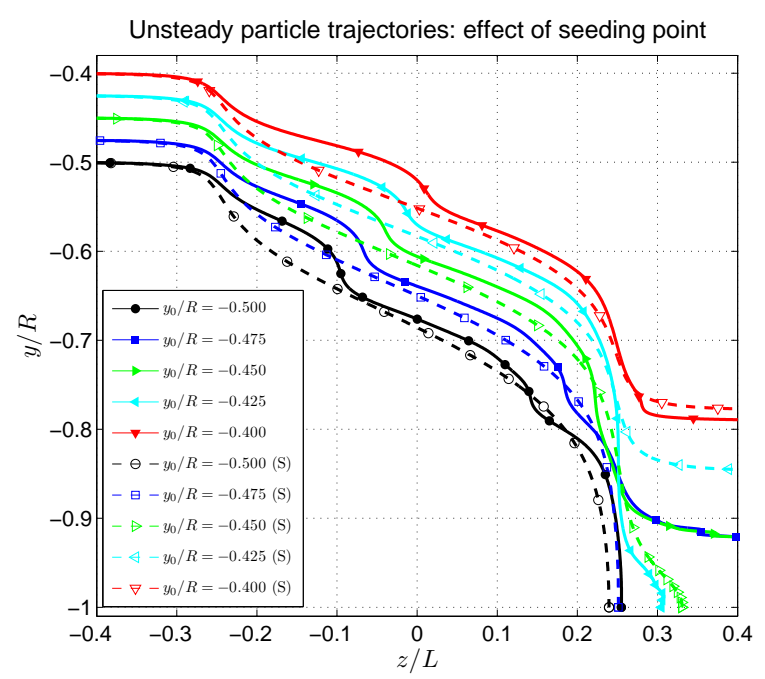

FIG. 6: (Color online) Exemplificative trajectories of magnetic particles seeded at $t_{0} / T=0.5$ [35]. Pulsatile flow conditions (solid lines with filled markers) are compared to steady ones (dashed lines with empty markers). Steady flow conditions are marked by symbol S in the legend.

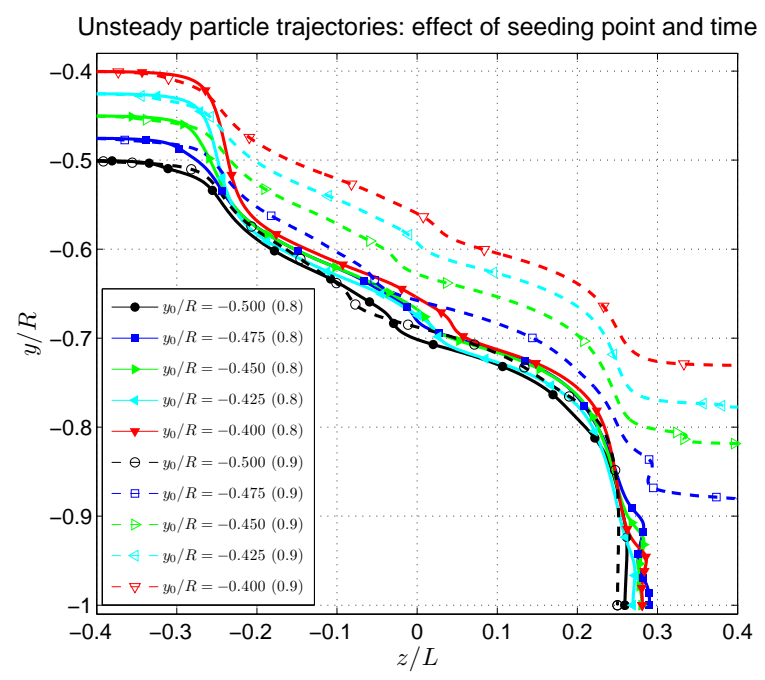

FIG. 7: (Color online) Exemplificative trajectories of magnetic particles seeded in pulsatile flow at $t_{0} / T=$ 0.8 (solid lines with filled markers) and 0.9 (dashed lines with empty markers). Non-dimensional seeding time is reported, in parentheses, in the legend.

located at frame origin was identified as a key parameter affecting capture efficiency [25]. For the above choice of parameters, $\mathrm{Mn}_{p}=0.005$ and, according to the scaling law obtained in [25], $\eta \approx$ 0.27; however, this figure should not be considered as a tight reference, since the magnetic source model used in [25] is slightly different from the one adopted in the present study. Capture efficiency was also assessed under the considered unsteady flow conditions, in particular by seeding 31397 
particles at the inlet section $\left(x_{0} / R\right)^{2}+\left(y_{0} / R\right)^{2}<1, z_{0} / L=-1$ (in practice, half-section was only considered, thanks to symmetry). Clearly, in the unsteady case the fraction of captured particles $\eta$ varies in general with the seeding time $t_{0}$, i.e. $\eta=\eta\left(t_{0}\right)$, and a period-averaged efficiency should be introduced, namely $\bar{\eta}:=T^{-1} \int_{0}^{T} \eta\left(t_{0}\right) d t_{0}$. However, in light of the fact that the present work mainly addresses fluidic modeling while using magnetic targeting as a valuable application field, we only evaluated $\eta$ for $t_{0} / T=k / 10$, with $k=0,1, \ldots, 9$; corresponding mean efficiency is 0.40 , with standard deviation 0.005. Despite the low variability of $\eta$ for the considered example, it is worth emphasizing the effect of unsteadiness. For instance, the inlet portion associated with magnetic particle capture is shadowed in Fig. 8, for given seeding times $t_{0} / T$. Time-variability of the curve separating capture/escape regions (which may be named "capture horizon" for magnetic targeting) should be noticed. Moreover, the occurrence of capture regions which are not simply connected domains (as for e.g. $t_{0} / T=0.2$ ) is a peculiar effect of unsteady targeting (this effect also occurs for $t_{0} / T=0.5$, consistently with the resulting trajectories in Fig. 6). Such an effect is trivially due to the fact that, for unsteady conditions, trajectories (i.e. pathlines) do not coincide with streaklines, so that two particles passing through a certain point of the fluid domain yet at different times are endawed, in general, with different velocities.

The aforementioned results regarding magnetic targeting do not make any claims of generality: they mainly serve to show the potential for effective applicability of the proposed analytical model for pulsatile flows. Indeed, by exploiting an accurate and efficient fluidic model it is possible to tackle complex, unsteady problems at an affordable cost, thus enabling a variety of further and deeper studies.

\section{CONCLUSIONS}

An exact solution to the inverse Womersley problem was derived for the fully-developed, laminar pulsatile flow of a viscous Newtonian fluid, within a cylindrical vessel with rigid walls. In particular, given a time-periodic flow rate, the axisymmetric velocity profile was obtained by means of two neat and computable maps relating the corresponding Fourier coefficients. Such maps were derived by solving the Poisson problems formulated in [20], provided that the considered problem admits solution as recently proved in [19]. As discussed in Sec. I, despite some degree of intrinsic simplification, fully developed flows represent a sort of natural test-case for both theoretical and experimental investigations, and their properties in the pulsatile case are object of ongoing research. In this context, the solution of the inverse problem deserves a special mention, since flow rate can be actually measured in many practical situations (contrarily to pressure gradient). 

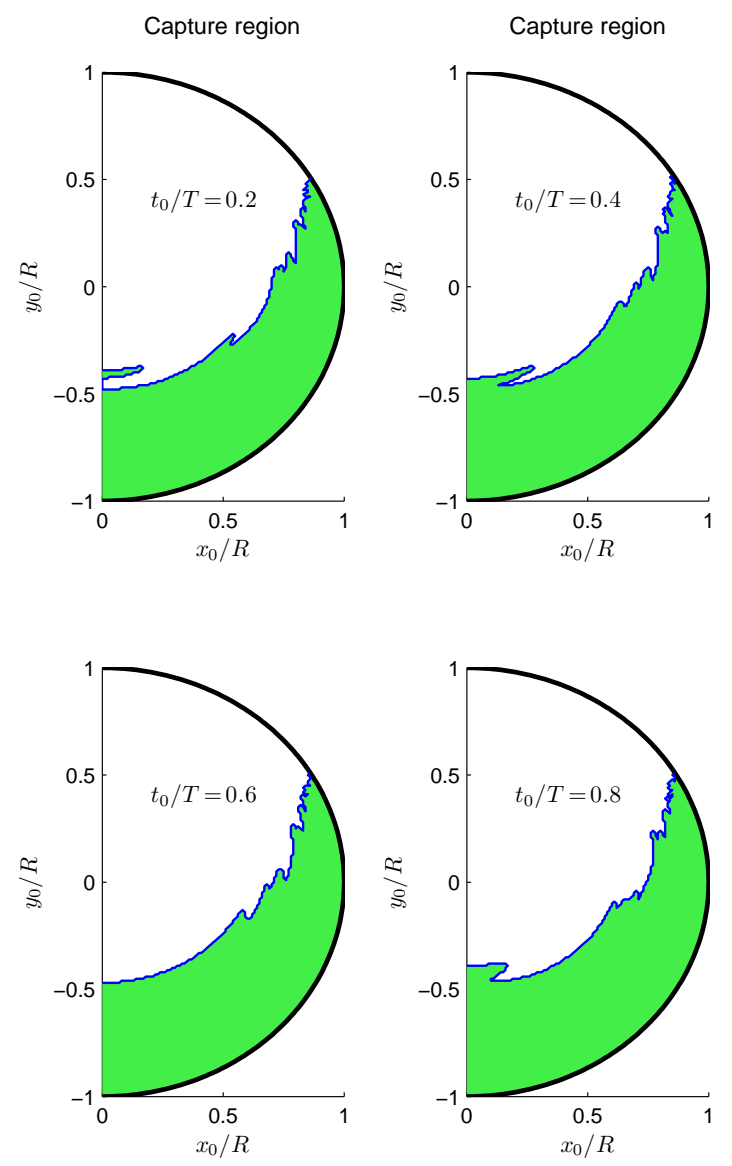

FIG. 8: (Color online) Exemplificative capture regions (shadowed) of the inlet cross-section, for the following non-dimensional seeding times $t_{0} / T:\{0.2,0.4,0.6,0.8\}$. Half-region is represented, due to symmetry with respect to the $y z$ plane $[36]$.

Hence, solution to the inverse Womersley problem represents a valuable tool/benchmark for a variety of research branches, also encompassing biological fluid dynamics (including targeted drug delivery) and biomedical engineering at large (e.g. for development of peristaltic pumps or biomedical intravascular devices for the human cardiovascular system). The main advantage of such an analytical solution compared to fully numerical approaches mainly resides in computational efficiency. Indeed, a generic finite element commercial solver may get to the same flow field solution by roughly taking a thousand times as long as a high-level code implementation of the provided maps. Such an asset was demonstrated in the present work, by considering both blood-like flows in larger/smaller human vessels and purely pulsatile flows, which can be of interest for the development of in vitro test-benches. Moreover, thanks to the aforementioned asset, the proposed solution was originally applied in the context of magnetic particle targeting, so as to highlight 
some peculiar effects due to pulsatility on particle trajectories and capture efficiency. Such a transport problem is increasingly drawing the attention of an interdisciplinary community, ranging from physicians to biomedical engineers, physicists and roboticists, thanks to its potential for targeted therapy. However, current approaches are essentially based on steady flows, which suitably applies to microvessels only. Nevertheless, short-/mid-term development of miniaturized interventional tools to be magnetically guided within body vessels [29] makes it necessary to also address larger flow domains, where pulsation plays a role. In such a context, by exploiting a classical magnetic force model also accounting for demagnetization effects and particle saturation, it was shown the relevance on targeting of particle seeding time (besides position in the cross-sectional plane), as well as the occurrence of not simply connected capture regions at vessel inlet, completely lacking in the steady case.

Such an exemplificative application does not make any claims of generality; however, it takes a preliminary step towards the effective exploitation of analytical, easily computable and physically relevant flow models for biomedical and microrobotic applications. In this spirit, physically-based values were assigned to all relevant parameters involved in the magnetic targeting problem, thus allowing for the description of a wide variety of real-life biomedical applications [25]. Moreover, with the modeling framework introduced in the present paper it is possible to tackle more refined targeting studies, for instance: (i) derivation of scaling laws for the capture efficiency in the unsteady case (in [25] such a study was carried out with respect to the non-dimensional parameter $\mathrm{Mn}_{p}$; other parameters like $\mathrm{Wo}_{R, N}$ are expected to play a role for unsteady flow conditions); (ii) identification of relevant non-dimensional parameters for more complete targeting problems involving extravasation (thus extending, for instance, the analysis carried out in [27]; also in this case $\mathrm{Wo}_{R, N}$ is expected to come into play). More in general, the proposed modeling approach is credited to hold potential for improving many current approaches dealing with pulsatile flows in cylindrical vessels, either focused on essentially fluidic effects or considering active transport/guidance of interventional agents within the pulsating fluid. In both cases, in fact, current approaches commonly determine the velocity field by scaling the (steady) parabolic solution by a factor accounting for flow rate variability (see e.g. [37, 38] for two-dimensional examples), thus introducing an approximation of the flow field which may be difficult to assess. Nevertheless, further generalizations of the proposed analytical approach can be envisaged. On regard, the basic hypothesis of fully-developed flow strongly supports analytical treatment and thus it may be relaxed at the cost of more involved derivations. Moreover, incorporation of non-Newtonian constitutive laws might be justified also in larger vessels (where pulsatility effects cannot be neglected), provided that higher shear-rates 
occur in some flow regions, e.g. due to partial obstructions. Both the aforementioned issues have been partly tackled in [39] for specific instances of the direct Womersley problem, thus supporting the potential interest in the inverse one as well. Furthermore, while considering a fully-developed flow of a Newtonian fluid, it may be of interest to extend the proposed approach to more generic cross-sections, starting from the elliptic one. A first step towards such an extension has been taken in [40]; corresponding analytic burden seems to be considerably increased with respect to the case of the circular cross-section. The aforementioned applications, as well as the many research lines envisaged for direct incorporation of the proposed analytical approach, further strengthen its potential for effective exploitation in an interdisciplinary context.

\section{Appendix: Magnetic field submodel}

Premise: without affecting consistency, in the present section we tolerate some abuse of notation with respect to the rest of the paper, for ease of presentation.

The magnetic field $\mathbf{H}$ of an axial, cylindrical permanent magnet having radius $r_{m}$ and length $l_{m}$ is classically modeled by exploiting the equivalent currents method (see e.g. [41]). Here we recall such model in order to provide readers with useful expressions, in view of practical applications (indeed, the considered expressions are prone to typos/misprints, even within very sound papers like e.g. [24]). Moreover, due to symmetry, we represent $\mathbf{H}$ in a cylindrical coordinate system $\left(\hat{\mathbf{e}}_{\rho}, \hat{\mathbf{e}}_{\theta}, \hat{\mathbf{e}}_{z}\right)$ having origin in the center of a cylinder base surface, with $\hat{\mathbf{e}}_{z}$ pointing outwards (conversion e.g. to the frame introduced in Sec. IIIB is straightforward). In such a frame, cylinder magnetization is assumed to be $M_{m}^{s} \hat{\mathbf{e}}_{z}$, where $M_{m}^{s}$ represents magnet saturation magnetization.

The considered magnetic field $\mathbf{H}=\mathbf{H}(\rho, z)$ reads:

$$
\mathbf{H}=\frac{M_{m}^{s}}{2 \pi} \int_{\xi=0}^{l_{m}}\left[h_{\rho}(\rho, z+\xi) \hat{\mathbf{e}}_{\rho}+h_{z}(\rho, z+\xi) \hat{\mathbf{e}}_{z}\right] \mathrm{d} \xi
$$

with

$$
\begin{gathered}
h_{\rho}(\rho, z):=\frac{1}{\sqrt{Q}} \frac{z}{\rho}\left[\frac{2-k^{2}}{2\left(1-k^{2}\right)} E(k)-K(k)\right], \\
h_{z}(\rho, z):=\frac{1}{\sqrt{Q}}\left[\frac{P}{Q} \frac{1}{1-k^{2}} E(k)+K(k)\right],
\end{gathered}
$$

where $Q:=\left(r_{m}+\rho\right)^{2}+z^{2}, k^{2}:=4 r_{m} \rho / Q$ and $P:=r_{m}^{2}-\rho^{2}-z^{2}$. Moreover, $K$ and $E$ respectively denote the (classical) complete elliptic integrals of the first and second kind, defined as follows:

$$
K(k):=\int_{\alpha=0}^{\pi / 2} \frac{\mathrm{d} \alpha}{\psi_{k}(\alpha)}, \quad E(k):=\int_{\alpha=0}^{\pi / 2} \psi_{k}(\alpha) \mathrm{d} \alpha,
$$


where $\psi_{k}(\alpha):=\sqrt{1-k^{2} \sin ^{2}(\alpha)}$. Furthermore, by recalling the fact that $k \mathrm{~d} K / \mathrm{d} k=E /\left(1-k^{2}\right)-$ $K$ and $k \mathrm{~d} E / \mathrm{d} k=E-K$, expressions (A.2) and (A.3) can be straightforwardly differentiated so as to obtain:

$$
\begin{gathered}
\beta \frac{\partial h_{\rho}(\rho, z)}{\partial \rho}=\sigma_{1} \gamma+\sigma_{4} h_{\rho}+\sigma_{7} \frac{h_{\rho}}{\rho}, \\
\beta \frac{\partial h_{\rho}(\rho, z)}{\partial z}=\beta \frac{\partial h_{z}(\rho, z)}{\partial \rho}=\sigma_{2} \gamma+\sigma_{5} h_{\rho}+\sigma_{8} \frac{h_{\rho}}{z}, \\
\beta \frac{\partial h_{z}(\rho, z)}{\partial z}=\sigma_{3} \gamma+\sigma_{6} h_{z},
\end{gathered}
$$

where $\quad \sigma_{1}:=3 z\left(r_{m}^{2}-\rho^{2}+z^{2}\right), \quad \sigma_{2}:=-6 \rho z^{2}, \quad \sigma_{3}:=-2 z\left(2 r_{m}^{2}-2 \rho^{2}+z^{2}\right), \quad \sigma_{4} \quad:=$ $\rho\left(3 r_{m}^{2}-2 \rho^{2}-3 z^{2}\right), \sigma_{5}:=z\left(r_{m}^{2}+\rho^{2}\right), \sigma_{6}:=z P, \sigma_{7}:=-\left(r_{m}^{2}+z^{2}\right)^{2}, \sigma_{8}:=\left(r_{m}^{2}-\rho^{2}\right)^{2}$, $\beta:=Q^{2}\left(1-k^{2}\right)$ and $\gamma:=2 r_{m}^{2} E(k) /\left[Q^{3 / 2}\left(1-k^{2}\right)\right]$. The above introduced expressions permit to analytically compute $\nabla \mathbf{H}$, as needed for computing the magnetic force model introduced in Sec. IIB.

Finally, it is worth remarking that, by recalling the asymptotic expansion of $K$ and $E$ for $k \rightarrow 0$, it is easy to circumvent the representation singularities associated with $\rho=0$ in the expressions (A.2), (A.5) and (A.6). Indeed, for $\rho \rightarrow 0$ (i.e. $\left.k^{2} \rightarrow 0\right), K=\pi / 2\left(1+k^{2} / 4+9 k^{4} / 64\right)+\mathrm{O}\left(k^{6}\right)$ and $E=\pi / 2\left(1-k^{2} / 4-3 k^{4} / 64\right)+\mathrm{O}\left(k^{6}\right)$, so that $h_{\rho} \approx \mu(z) \rho$, with $\mu(z):=\left(3 \pi r_{m}^{2} / 2\right) z /\left(r_{m}^{2}+z^{2}\right)^{5 / 2}$, and $h_{\rho} / \rho \approx \mu(z)$. These relations prove to be useful for the numerical implementation of the magnetic field model at hand, namely when computing $\mathbf{H}$ at points having $\rho<\bar{\rho}$, where $\bar{\rho}$ is a predefined threshold (a practical alternative may consist in chopping the radial coordinate below such a threshold).

\section{Acknowledgments}

The work of P. Miloro and A. Menciassi was partially supported by Fondazione Cassa di Risparmio di Pisa, within the framework of the Micro-VAST (Microsystems for VAscular diagnosticS and inTervention) project.

[1] G. K. Batchelor, An introduction to fluid dynamics, Cambridge Mathematical Library (Cambridge University Press, Cambridge, 2000).

[2] T. Sexl, Z. Physik 61, 349 (1930). 
[3] J. R. Womersley, J. Physiol. 127, 553 (1955).

[4] S. Uchida, Z. Angew. Math. Phys. 7, 403 (1956).

[5] P. D. Verma, P. Indian A.S. - Math. Sci. 26, 282 (1960).

[6] P. D. Verma, P. Indian A.S. - Math. Sci. 26, 447 (1960).

[7] G. Pontrelli, Comput. Fluids 27, 367 (1998).

[8] S. Tsangaris and N. W. Vlachakis, Z. Angew. Math. Phys. 54, 1094 (2003).

[9] S. McGinty, S. McKee, and R. McDermott, J. Non-Newton. Fluid 162, 54 (2009).

[10] L. Formaggia, A. Quarteroni, and A. Veneziani, in \emph\{\bibinfo\{booktitle\}\{Complexsystemsinbiomedicine\} (Springer, Milan, 2006) pp. 243-287.

[11] D. Valassis, R. Dodde, B. Esphuniyani, J. Fowlkes, and J. Bull, Biomed. Microdevices (to be published, DOI:10.1007/s10544-011-9591-x).

[12] Y. Hoi, B. A. Wasserman, Y. Y. J. Xie, S. S. Najjar, L. Ferruci, E. G. Lakatta, G. Gerstenblith, and D. A. Steinman, Physiol. Meas. 31, 291 (2010).

[13] R. Ponzini, C. Vergara, G. Rizzo, A. Veneziani, A. Roghi, A. Vanzulli, O. Parodi, and A. Redaelli, IEEE T. Bio-Med. Eng. 57, 1807 (2010).

[14] S. Tsangaris and N. Stergiopulos, J. Biomech. 21, 263 (1988).

[15] D. Das and J. Arakeri, J. Appl. Mech. 67, 274 (2000).

[16] K. Haddad, O. Ertunc, M. Mishra, and A. Delgado, Phys. Rev. E 81, 016303 (2010).

[17] S. Ray, B. Unsal, F. Durst, O. Ertunc, and O. Bayoumi, J. Fluid. Eng. - T. ASME 127, 405 (2005).

[18] G. P. Galdi, An introduction to the mathematical theory of the Navier-Stokes equations. Vol. I, Springer Tracts in Natural Philosophy, Vol. 38 (Springer, New York, 1994).

[19] H. Beirão da Veiga, Arch. Ration. Mech. An. 178, 301 (2005).

[20] G. P. Galdi and A. M. Robertson, J. Math. Fluid Mech. 7, S215 (2005).

[21] E. P. Furlani and K. C. Ng, \bibfield\{journal\}\{\bibinfo\{journal\}\{Phys.Rev.E\} $\} \backslash$ textbf $\{\backslash$ bibinfo\{volume $\{$ 73\}\}, \\bibinfo\{pages $\}$ 061919\} (\bibinfo\{year\}\{2006\}).

[22] E. P. Furlani, Y. Sahoo, K. C. Ng, J. C. Wortman, and T. E. Monk, Biomed. Microdevices 9, 451 (2007).

[23] E. J. Furlani and E. P. Furlani, J. Magn. Magn. Mater. 312, 187 (2007).

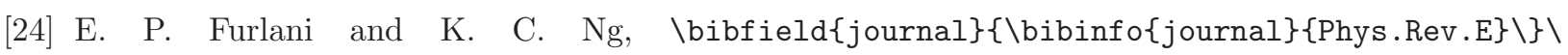
textbf $\{\backslash$ bibinfo\{volume $\{777\}\}, \backslash \backslash$ bibinfo\{pages $\}\{061914\}$ ( \bibinfo\{year\}\{2008\}).

[25] J. W. Haverkort, S. Kenjeres, and C. R. Kleijn, Phys. Rev. E 80, 016302 (2009).

[26] S. Shaw, P. V. S. N. Murthy, and S. C. Pradhan, J. Magn. Magn. Mater. 322, 1037 (2010).

[27] A. Nacev, C. Beni, O. Bruno, and B. Shapiro, J. Magn. Magn. Mater. 323, 651 (2011).

[28] J.-B. Mathieu and S. Martel, Magnet. Reson. Med. 63, 1336 (2010).

[29] B. J. Nelson, I. K. Kaliakatsos, and J. J. Abbott, Annu. Rev. Biomed. Eng. 12, 55 (2010).

[30] L. Landau and E. Lifshitz, Fluid Mechanics, Course of Theoretical Physics, Vol. 6 (Pergamon Press, London, 1959). 
[31] H. Lamb, Hydrodynamics, Cambridge Mathematical Library (Cambridge University Press, Cambridge, 1993).

[32] J. Krejza, M. Arkuszewski, S. E. Kasner, J. Weigele, A. Ustymowicz, R. W. Hurst, B. L. Cucchiara, and S. R. Messe, Stroke 37, 1103 (2006).

[33] G. S. Kassab, Am. J. Physiol. - Hearth C. 290, H894 (2006).

[34] See Supplemental Material at [URL: tbd] for an animation of the flow field associated with TC-4b.

[35] See Supplemental Material at [URL: tbd] for an animation of the trajectories reported in Fig. 6, also showing the underlying flow field.

[36] See Supplemental Material at [URL: tbd] for an animation of the whole capture region exemplified in Fig. 8, also showing the underlying flow field.

[37] L. Arcese, M. Fruchard, F. Beyeler, A. Ferreira, and B. Nelson, in Proceedings of ICRA 2011, Shanghai, China (2011) pp. 4121-4126.

[38] A. Gizzi, M. Bernaschi, D. Bini, C. Cherubini, S. Filippi, S. Melchionna, and S. Succi,

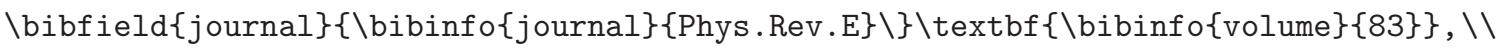
bibinfo\{pages\}\{031902\} ( \bibinfo\{year $\}\{2011\}$ ).

[39] S. Siddiqui, N. Verma, S. Mishra, and R. Gupta, Appl. Math. Comput. 210, 1 (2009).

[40] S. Gupta, D. Poulikakos, and V. Kurtcuoglu, Phys. Fluids 20, 093607 (2008).

[41] E. P. Furlani, Permanent Magnet and Electromechanical Devices (Academic Press, London, 2001). 